\section{Ausländische Ärzte in Deutschland - Anerkennung ausländischer Studienabschlüsse, Weiterbildungszeiten und im Ausland abgeschlossener Facharztweiterbildungen}

\section{Einführung}

Bis 2016 ist die Zahl der ausländischen Ärztinnen und Ärzte in Deutschland auf ca. 46.700 angestiegen; damit stellen ausländische Berufsträger 10,6\% der in Deutschland gemeldeten Ärzteschaft. ${ }^{1}$ Von diesen besitzen 2017 insgesamt 11,8\% eine ausländische Staatsbürgerschaft. ${ }^{2}$ Medizinerinnen und Mediziner mit Abschlüssen aus anderen Ländern müssen, ebenso wie ihre in Deutschland ausgebildeten Kolleginnen und Kollegen, eine Approbation oder Berufserlaubnis nachweisen können und ihre ausländische Facharztausbildung in Deutschland anerkennen lassen. Auf dem verhältnismäßig schnellsten und einfachsten Weg ist dies Ärztinnen und Ärzten aus Mitgliedstaaten der Europäischen Union möglich. Für Ärzte aus sogenannten Drittstaaten gestaltet sich das Unterfangen, eine Approbation zu erhalten, schwieriger.

Während die Vorschriften über die ärztliche Approbation in der Bundesärzteordnung (BÄO) und der Approbationsordnung (ÄApprO) bundesweit einheitlich gelten, sind die Bestimmungen über die Weiterbildung in den Heilberufskammergesetzen (Ländergesetze) und autonomen Satzungen der Landesärztekammern geregelt. Insbesondere die Vorschriften über die Anerkennung der Facharztausbildungen und die Ausbildungszeiten in den Ländergesetzen sind inhomogen, im Wesentlichen jedoch an der Muster-Weiterbildungsordnung (MWBO) der Bundesärztekammer angelehnt. Aufgrund der vielfältigen Regelungen in unterschiedlichen Gesetzen gestalten sich die Voraussetzungen zur Ausübung der ärztlichen Tätigkeit äußerst

1 Gesundheitsdaten - Mehr ausländische Ärzte im ambulanten Bereich 2016, aufrufbar unter: http://gesundheitsdaten.kbv.de/cms/html/ 17 078.php (29.11.2018).

2 Statistik Bundesärztekammer Ausländische Ärztinnen und Ärzte 2017, abrufbar unter: https://www.bundesaerztekammer.de/ ueber-uns/aerztestatistik/aerztestatistik-2017/ auslaendische-aerztinnen-und-aerzte/ (20.09.2018). komplex und sind mithin für mögliche Bewerber schwer zu überblicken.

\section{Anerkennung ausländischer Studienabschlüsse und Berufserlaubnis}

Ärztinnen und Ärzten, die eine nach 1976 abgeschlossene ärztliche Ausbildung in einem der Mitgliedstaaten der Europäischen Union oder in einem anderen Vertragsstaat des Abkommens über den Europäischen Wirtschaftsraum nachweisen können, wird im Verfahren der automatischen Anerkennung die Approbation erteilt. ${ }^{3}$ Die Ausbildung, einschließlich der ärztlichen Prüfung, gilt dabei als der deutschen ärztlichen Ausbildung vergleichbar, wenn sie durch Vorlage eines Europäischen Berufsausweises nachgewiesen wird, §3 Abs. 1 BÄO. Bei später hinzugetretenen Mitgliedstaaten ist gegebenenfalls auf den jeweiligen Beginn der Ausbildung nach dem Beitritt des Staates abzustellen. Eine individuelle Gleichwertigkeitsprüfung ist nicht erforderlich.

Sofern Ärztinnen und Ärzte über Ausbildungsnachweise, die in einem Drittstaat ausgestellt wurden, verfügen, müssen diese zum Erhalt der Approbation die Gleichwertigkeit ihres Ausbildungsstandes nachweisen, § 3 Abs. 3 BÄO. Die Kenntnisprüfung bezieht sich ausdrücklich auf den Inhalt des deutschen Studiums, § 3 Abs. 3 S. 3 BÄO. ${ }^{4}$ Ärztinnen und Ärzte mit Abschlüssen aus Drittstaaten müssen in dieser Prüfung grundsätzlich nachweisen, dass sie über das Wissen verfügen, das von einheimischen Absolventen medizinischer Hochschulen verlangt wird. ${ }^{5}$ Der Schwerpunkt der Prüfung liegt auf den Fächern Innere Medizin und Chirurgie. Maßstab für die zu prüfende

3 Art. 21 EU-Richtlinie 2005/36/EG.

4 OVG NRW, Az. 13 A 235/15, Urt. v. 17.02.2017, Rn. 43.

5 BVerwG, Az. 3 C 33/07, Urt. v. 11.12.2008 Rn. $18 \mathrm{f}$.

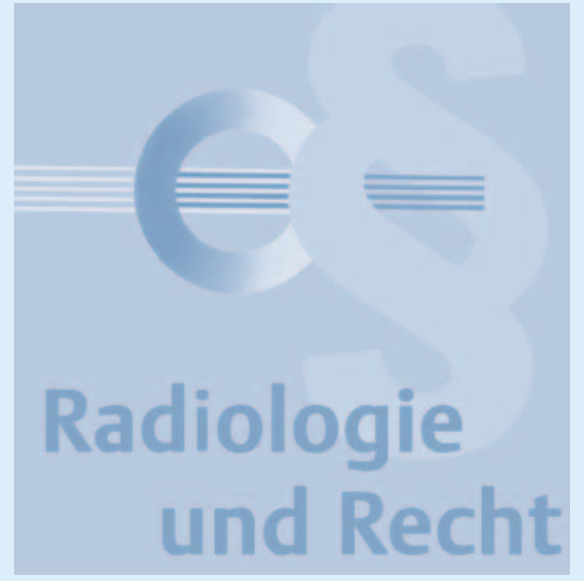

Gleichwertigkeit ist der Ausbildungsstand nach einem Studium der Medizin von mindestens sechs Jahren in der Bundesrepublik Deutschland (§3 Abs.1 S. 1 Nr. 4 BÄO), von denen mindestens acht, höchstens zwölf Monate auf eine praktische Ausbildung in Krankenhäusern oder geeigneten Einrichtungen der ärztlichen Krankenversorgung entfallen.

Der Ausbildungsstand ist als gleichwertig anzusehen, wenn keine wesentlichen Unterschiede gegenüber der deutschen Ärzteausbildung vorliegen. Solche Unterschiede lägen beispielsweise dann vor, wenn die nachgewiesene Ausbildungsdauer die deutsche Ausbildungsdauer mindestens um ein Jahr unterschreitet oder sich die Ausbildungsfächer wesentlich von der deutschen Ausbildung unterscheiden. ${ }^{6}$ Es besteht jedoch die Möglichkeit, wesentliche Unterschiede ganz oder teilweise durch Kenntnisse auszugleichen, die der Antragsteller im Rahmen seiner ärztlichen Berufspraxis erworben hat. ${ }^{7}$ Dabei ist nicht entscheidend, in welchem Staat der Antragsteller die Kenntnisse und Fähigkeiten erworben hat, §3 Abs. 2. S. 5 BÄO. Der Nachweis über den Ausgleich der festgestellten wesentlichen Unterschiede ist durch Bestehen einer sogenannten Eignungsprüfung („Defizitprüfung“) zu erbringen. ${ }^{8}$

In allen anderen Fällen können Drittstaatsangehörige nur eine Berufserlaubnis bean-

6 BVerwG, Az. 3 B 46/05, Urt. v. 22.09.2005, Rn. 2.

7 VG Köln, Az. 7 K 2719/15, Urt. v. 22.0.8.2017, Rn. $39 \mathrm{ff}$.

8 VG Köln, Az. 7 K 4496/13, Urt. v. 22.09.2015, Rn. 40. 
tragen, die zur Ausübung des ärztlichen Berufs für eine befristete Zeit ermächtigt, $\S 10 \mathrm{BÄO}$. Allerdings ist eine Beantragung nur dann möglich, wenn auch eine Aufenthalts- und Arbeitserlaubnis vorgelegt wird. Antragstellern, die über einen Ausbildungsnachweis als Arzt verfügen, der in einem Mitgliedstaat der Europäischen Union ausgestellt wurde, wird in der Regel keine Berufserlaubnis erteilt, da diesen ohnehin ein Anspruch auf Erteilung der Approbation zusteht. Abweichend davon kann auf Antrag die Erlaubnis erteilt werden, soweit „im Hinblick auf die beabsichtigte ärztliche Tätigkeit ein besonderes Interesse an der Erteilung der Erlaubnis besteht." 9

Die Erteilung der Berufserlaubnis erfolgt widerruflich und wird nur bis zu einer Gesamtdauer der ärztlichen Tätigkeit von höchstens zwei Jahren erteilt. Hintergrund der Befristung ist, dass nach dem Willen des Gesetzgebers alle Antragsteller umgehend die Approbation beantragen sollen und das Approbationsverfahren vorrangig betrieben werden soll. Die Behörde kann die Berufserlaubnis sowohl inhaltlich als auch örtlich auf eine Beschäftigungsstelle beschränken. ${ }^{10}$ Anders als bei der Approbationserteilung ist für die Erteilung der Berufserlaubnis die Feststellung der Gleichwertigkeit nicht erforderlich. Bei Vorliegen eines Zeugnisses über den Abschluss des Medizinstudiums steht es im Ermessen der Behörde, die Berufserlaubnis zu erteilen. Sofern allerdings keine gewichtigen Interessen gegen die Erteilung der Berufserlaubnis sprechen und alle Tatbestandsvoraussetzungen vorliegen, wird die Behörde dem Antrag stattgeben.

Die Bundesärzteordnung und die Approbationsordnung für Ärzte enthalten zusätzlich zu den Regelungen zur automatischen Anerkennung bzw. Prüfung der Gleichwertigkeit der Ausbildung auch detaillierte Regelungen über den Ablauf des Anerkennungsverfahrens, insbesondere hinsichtlich der vorzulegenden Unterlagen, der Entscheidungsfristen und des Inhalts der Bescheide. Neben der amtlich beglaubigten Kopie der Befähigungsnachweise oder der Unbedenklichkeitsbescheinigung durch das Herkunftsland

9 Vgl. § 10 Abs. 1a BÄO.

10 BT-Drs. 18/1162, S. 18. wird unter anderem eine tabellarische Aufstellung der absolvierten Ausbildungsgänge gefordert. ${ }^{11}$ Ausländische Ärztinnen und Ärzte haben, wie auch deutsche Ärztinnen und Ärzte, darüber hinaus die allgemeinen Vorgaben für die Beantragung einer Approbation und die dazu erforderlichen Unterlagen nach § 39 ÄApprO zu beachten.

\section{Anerkennung von Fortbildungszeiten und abgeschlossenen Facharztausbildungen bei
ausländischen Ärzten}

Ursprünglich war die Anrechnung von Fortbildungszeiten nicht erst nach dem Erhalt der Approbation, sondern bereits ab Vorliegen einer Berufserlaubnis möglich. Dieser Zusatz wurde jedoch von vielen Ärztekammern in ihren Weiterbildungsordnungen gestrichen: ${ }^{12}$

Die Beschränkungen der Ärztekammern, Fortbildungszeiten nur noch nach dem Erhalt der Approbation bei ausländischen Ärzten gelten zu lassen, basiert auf der Umsetzung der EU-Richtlinie (Richtlinie 2013/55/EU) vom 20.11.2013 zur Änderung der Richtlinie 2005/36/EG über die Anerkennung von Berufsqualifikationen und der Verordnung (EU) Nr. 1024/2012 über die Verwaltungszusammenarbeit mithilfe des Binnenmarkt-Informationssystems („IMIVerordnung“). In der Richtlinie 2005/36/EG sind die Mindestanforderungen an die ärztliche Grundausbildung (Art. 24) sowie an die fachärztliche Weiterbildung (Art. 25) normiert, die durch die Richtlinie 2013/55/EU modifiziert wurden.

In Art. 25 Abs. 1 der Neufassung heißt es dazu wie folgt:

„Die Zulassung zur fachärztlichen Weiterbildung setzt voraus, dass eine ärztliche Grundausbildung nach Artikel 24 Absatz 2 abgeschlossen und als gültig anerkannt worden ist, mit der angemessene medizinische Grundkenntnisse erworben wurden."

11 Näheres in §3 Abs. 6 BÄO.

12 Exemplarisch: Weiterbildung nur mit Approbation, aufrufbar unter http://www.laek-rlp.de/ aerzteservice/weiterbildung/weiterbildungnur-mit-approbation.php (29.11.2018).
Artikel 24 Abs. 2 der Neufassung legt den Umfang der ärztlichen Grundausbildung fest:

„Die ärztliche Grundausbildung umfasst mindestens fünf Jahre (kann zusätzlich in der entsprechenden Anzahl von ECTS-Punkten ausgedrückt werden) und besteht aus mindestens 5500 Stunden theoretischer und praktischer Ausbildung an einer Universität oder unter Aufsicht einer Universität.

Bei Berufsangehörigen, die ihre Ausbildung vor dem 1. Januar 1972 begonnen haben, kann die in Unterabsatz 1 genannte Ausbildung eine praktische Vollzeitausbildung von sechs Monaten auf Universitätsniveau unter Aufsicht der zuständigen Behörden umfassen. “

Ziel der Novellierung war es, ein gleichbleibend hohes Niveau der öffentlichen Gesundheit und Patientensicherheit in der Europäischen Union zu gewährleisten, indem Kriterien der ärztlichen Grundausbildung und fachärztlichen Weiterbildung festgelegt und ein entsprechender Mindeststandard sichergestellt werden. ${ }^{13}$

Für die Zulassung zur Weiterbildung bedarf es nach Art. 25 Abs. 1 der EU-Richtlinie demzufolge einer abgeschlossenen und als gültig anerkannten Grundausbildung. Fraglich ist, ob die Berufserlaubnis nach $\S 10$ BÄO den in Art. 25 neue Fassung definierten Mindeststandard erfüllt. §10 Abs. 1 BÄO legt fest, dass die Berufserlaubnis auf Antrag Personen erteilt werden kann, die „eine abgeschlossene Ausbildung für den ärztlichen Beruf nachweisen." Anders als bei der Approbationserteilung ist die Behörde jedoch nicht an die strengen Voraussetzungen des $\S 3$ BÄO und des Art. 24 der Neufassung der EU-Richtlinie gebunden. Das bedeutet auch, dass die Behörde weder die gesundheitliche Eignung des Antragstellers noch die Dauer des Medizinstudiums und der anschließenden praktischen Ausbildung überprüft bzw. überprüfen kann. Zwar liegt auch bei einer erteilten Berufserlaubnis ein abgeschlossenes Studium vor, dieses entspricht jedoch nicht zwingend den in Art. 24 der Neufassung der EU-Richtlinie normierten Anforderungen. Erst die Approbation stellt damit

13 Richtlinie 2013/55/EU, Erwägungsgrund Nr. 25. 
ein gültiges (dauerhaftes) Anerkenntnis im Sinne des Art. 25 dar.

Ein weiteres Hindernis für Bewerber stellt das Anerkennungserfahren von Fortbildungszeiten und Facharztausbildungen dar, dieses ist auf Bundesebene nicht einheitlich geregelt. Die Länder, denen gemäß Art. 74 Abs. 1 Nr. 19 Grundgesetz die Gesetzgebungskompetenz für das Gebiet der Heilberufe zusteht, mussten, um die verbindlichen europäischen Regelungen des Art. 24 der Europäischen Richtlinie in das deutsche Recht zu implementieren, ihre Heilberufsgesetze ändern. Für Deutschland sind in allen Angelegenheiten ärztlicher Weiterbildung die Landesärztekammern als Körperschaften des öffentlichen Rechts zuständig. Die von der Bundesärztekammer erarbeitete (Muster-) Weiterbildungsordnung, die Regelungen zur Anerkennung im Ausland abgeschlossener Facharztausbildungen enthält, hat dementsprechend lediglich empfehlenden Charakter. In die aktuellen Weiterbildungsordnungen der Länder wurde durch die zuständigen Landesärztekammern der Zusatz aufgenommen, dass Fortbildungszeiten erst nach Erteilung der Approbation oder nach Berufserlaubnis unter vorheriger Feststellung der Gleichwertigkeit der ärztlichen Grundausbildung angerechnet werden können, vgl. §5 Abs. 1 S. 1 Weiterbildungsordnung der Ärztekammer Niedersachsen.

Daher müssen die Antragstellenden in der Regel das Verfahren zur Erteilung der Approbation erfolgreich durchlaufen, bevor die Anerkennung der Fortbildungszeiten aufgrund einer im Ausland begonnenen Weiterbildung möglich ist. Dabei sollte jedoch nicht in Vergessenheit geraten, dass Tätigkeitszeiten nach der Erteilung der Berufserlaubnis im Inland ebenfalls als Weiterbildungszeiten anerkannt werden können und werden, sofern der Arbeitgeber über eine Weiterbildungsbefugnis verfügt. Über die Anerkennung bislang abgeleisteter Weiterbildungszeiten entscheidet die zuständige Ärztekammer.

Die vorstehend genannten Regelungen gelten weitestgehend auch für die Anerkennung im Ausland abgeschlossener Facharztausbildungen. Bevor die Anerkennung der Facharztausbildung möglich ist, muss der Antragsteller das Verfahren zur Erteilung der Approbation erfolgreich durchlaufen haben. Grundsätzlich muss für eine Anerkennung des Weiterbildungsnachweises die Gleichwertigkeit des Weiterbildungsstandes nachgewiesen werden. ${ }^{14}$ Das Erfordernis der Feststellung der Gleichwertigkeit ist unabhängig von der Staatsangehörigkeit, was bedeutet, dass auch Antragsteller aus EU-Mitgliedstaaten nach den Weiterbildungsordnungen der Landesärztekammern die Gleichwertigkeit des absolvierten Weiterbildungsgangs nachweisen müssen.

\section{Fazit}

Der Trend des Auseinanderdriftens von Behandlungsbedarf und Behandlungskapazitäten setzte sich in den vergangenen Jahren fort - die Schere öffnet sich weiter. ${ }^{15}$ Trotz der steigenden Zahl der Berufsträger (von 2015 bis 2016 Steigerung um ca. 10.000 auf $496.240^{16}$ ) greift mancherorts - insbesondere in ländlichen und strukturschwachen Regionen - weiterhin der (Fach-) Ärztemangel um sich. Die sich derzeit in Ausbildung befindende Generation deutscher Medizinerinnen und Mediziner stellt gesteigerte Anforderungen an die ausgewogene Work-Life-Balance und orientiert sich eher in Richtung der Ballungsgebiete größerer Städte mit attraktivem Umfeld, viele Landarztsitze werden nicht nachbesetzt werden können. Fraglich bleibt jedoch, ob diese Lücke in der ärztlichen Versorgung durch ausländische Ärzte geschlossen werden kann.

Die größten Schranken für deren ärztliche Betätigung errichten die Ärztekammern selbst: Große Schwierigkeiten im Zuge der Erlangung einer deutschen Zulassung bereiten ausländischen Antragstellern der Nachweis der Gleichwertigkeit der ärztlichen Ausbildung im Heimatland und der Nachweis

14 Vgl. § 10 Weiterbildungsordnung der Ärztekammer Niedersachsen.

15 Stellungnahme der Bundesärztekammer zur Ärztestatistik 2016: „Die Schere zwischen Behandlungsbedarf und Behandlungskapazitäten öffnet sich“, abrufbar unter: https://www. bundesaerztekammer.de/presse/pressemitteilungen/news-detail/die-schere-zwischenbehandlungsbedarf-und-behandlungskapazitaeten-oeffnet-sich/ (26.09.2018).

16 Gesundheitsdaten - Mehr als 496000 Ärzte in Deutschland, aufrufbar unter: http://gesundheitsdaten.kbv.de/cms/html/17 077.php (29.11.2018) ausreichend vorhandener Sprachkenntnisse sowie des Bestehens der verpflichtenden Kenntnisprüfungen. Eine mögliche Absenkung dieser Hürden ist jedoch - selbst in Zeiten akuten Ärztemangels - schwer zu rechtfertigen. Ohne eine gleichwertige Ausbildung ausländischer Bewerber ist eine Integration in das deutsche Gesundheitswesen und damit den Arbeitsmarkt nicht zu gewährleisten. Die Ärztekammern sind zudem verpflichtet, unter den ihnen angehörenden Ärzten eine - am Standard des in Deutschland ausgebildeten Mediziners gemessene - gleichbleibend hohe Qualität der ärztlichen Berufsausübung zu gewährleisten. Dafür sind die Abfrage der fachspezifischen Kenntnisstände der Bewerber und deren Sprachkenntnisse ein probates Mittel, letzteres insbesondere vor dem Hintergrund der immensen Bedeutung von Anamnese, Aufklärungs- und Therapiegesprächen im medizinischen Alltag. Auch die Überprüfung der Gleichwertigkeit von ausländischen Fortund Weiterbildungen im Hinblick auf ihre Dauer ist zur dauerhaften Sicherung eines hohen medizinischen Standards in Deutschland unabdingbar.

Insbesondere vor dem Hintergrund erheblicher Rechtsunsicherheiten im Zuge der Anerkennung der Qualifikation ausländischer Ärzte wird deutlich ${ }^{17}$ : Allein eine Steigerung der Beschäftigung ausländischer Ärztinnen und Ärzte im deutschen Gesundheitssystem wird jedenfalls nicht die Lösung des systemischen Problems des mittlerweile chronischen deutschen (Land-) Ärztemangels sein.

René T. Steinhäuser

Rechtsanwalt

Annika Stöbener

Wissenschaftliche Mitarbeiterin

Rechtsanwälte Wigge

Großer Burstah 42

20457 Hamburg

Telefon: (040) 3398705 - 90

Telefax: (040) 3398705 - 99

E-Mail: kanzlei@ra-wigge.de www.ra-wigge.de

17 BT-Drs. 18/11513, S. $10 \mathrm{f}$ 